\title{
Obesity in an ageing population: a proposed multidisciplinary intervention model for supporting cognitive performance and physical function in obese seniors
}

\begin{abstract}
Obesity in ageing adults is a precursor for increased risk of various diseases and chronic health conditions including cognitive impairment and poorer mobility thereby threatening quality of life and independent living for many seniors. Fortunately, weight loss and weight management interventions have been recognized as a means to help improve both physical function and cognition in obese older persons. Based on the literature, we propose that programs and services embodying a multidisciplinary team who focus on education and training related to physical activity, diet, and pharmaceuticals be offered to optimally support weight loss, improve mobility and enhance cognition for this population. Future intervention research is warranted to test this multidisciplinary approach in order to help develop suitable interventions and preventative measures to reduce or delay decline in mobility and the onset of cognitive impairment and dementia in obese seniors.
\end{abstract}

Keywords: obesity, older adults, cognitive performance, physical function
Volume 6 Issue 5 - 2017

\author{
Amanda Baker,' Amberly Baker ${ }^{2}$ \\ 'University of Ottawa, Canada \\ ${ }^{2}$ Bruyère Academic Family Health Team, Bruyère Continuing \\ Care, Canada
}

\begin{abstract}
Correspondence: Amanda Baker, School of Psychology, University of Ottawa, I 36 Jean-Jacques Lussier St., Canada KIN
\end{abstract} 6N5, Email amandaleigh.baker@gmail.com

Received: May 08, 2017 | Published: June 02, 2017

\section{Introduction}

Older adults $(>65)$ are the fastest growing age group in North America. ${ }^{1}$ In fact, the latest Canadian census ${ }^{2}$ revealed that for the first time, seniors over the age of 65 exceed the number of children under the age of 15 and this shift in demographics is projected to radically accelerate over the next two decades. ${ }^{3}$ While growth in older adult populations endures, public health and public policy rely on the health status of seniors to inform and determine their current and future service needs. The current brief review summarizes the risks associated with obesity in older adults and then diverts its focus to the cognitive and physical consequences. We also outline three key recommendations to consider for future prevention and intervention programs that explicitly target obese and overweight seniors.

By and large, obesity remains a primary health concern given that close to $70 \%$ of American adults are obese or overweight. 4,5 Consequently, the prevalence of obesity increases the risk of additional epidemic developments. For instance, obesity in older adults is welldocumented as a precursor for increased risk of various diseases and chronic health conditions such as cardiovascular disease, stroke, hypertension, type II diabetes, osteoporosis, some cancers, sleep apnea, anxiety and depression. ${ }^{6,7}$ In addition, obesity is a principal cause of disability in older adults. ${ }^{89}$ Changes in cognition and mobility are deemed the two most common and intertwined complexities faced by seniors ${ }^{10,11}$ and although these functions tend to gradually decline with the process of ageing, some studies reveal that they may deteriorate faster in older obese persons..$^{12}$ According to the literature, obesity is indeed associated with poorer mobility, stability and gait movement among older adults. ${ }^{13}$ Obesity can also contribute to cognitive deficits including diminished memory, decreased problem solving abilities and impaired decision making. ${ }^{14}$ Midlife obesity is also a risk factor for dementia in later years. ${ }^{15}$ Overall, evidence suggests that obesity can cause severe and widespread health problems among the rapidly ageing population thereby resulting in negative consequences on quality of life and independent living among seniors.

With the considerable growth rate and substantial increase in obesity among this population, a significant decline in cognitive performance and physical mobility will likely bring about high demands for general health care, home care and continuing care particularly in Canada. These adverse effects instigated by obesity parameters (e.g., body mass index (BMI), percentage of body fat, waist circumference) are therefore more relevant than ever as the population ages. Fortunately, intervention trials have been recognized as a means to help improve physical function ${ }^{16,17}$ and cognition ${ }^{18}$ in obese older persons.

Villareal et al., ${ }^{16}$ found that after a 52 -week intervention, both physical activity and diet improved obese older adults' physical function compared to either the physical activity or diet intervention alone. In addition, a recent systematic review and meta-analysis ${ }^{19}$ revealed that the most effective weight loss and weight management programs for obese individuals were those that combined physical activity and diet strategies. Furthermore, a review by Kramer \& Erickson ${ }^{20}$ which comprised of epidemiological observational studies and randomized human clinical interventions found that, in general, physical activity enhanced cognition and protected against the development of neurodegenerative diseases in obese older adults. However, they also noted that investigations of the benefits of physical activity programs to help reverse cognitive degeneration in obese older adults are still underdeveloped. Finally, geriatric research suggests that regular medication reviews by a health care professional may also aid with weight loss and improvements in cognition and physical function in obese older adults..$^{21}$ For example, some medications used by ageing adults are known to have adverse effects including weight gain (e.g., 
antipsychotics) and changes in cognition and behaviour (e.g., pain relief medication), all of which may impede their ability to participate in programs that focus on weight loss and weight management. On the other hand, some drugs can increase adherence to behaviour change and may improve physical functioning. ${ }^{21}$ Hence, medication reconciliation may help practitioners recognize and understand the influence of drugs and drug combinations in obese seniors that impact the efficacy of weight intervention programs.

Overall, obesity intervention and prevention programs are receiving a lot of attention and continue to be developed and tested as the need arises. Based on the current review of obesity and geriatric literatures, we emphasize three key components as the minimal requirement to optimally address the issues of cognitive decline and poor physical function in obese and overweight ageing adults. We propose that a multidisciplinary team with a focus on education and training related to physical activity, diet and pharmaceuticals would be ideal. Given that older adults are the least physically active group ${ }^{22}$ and are more likely to encounter additional complications from medications, weight loss and weight management services should examine the effects of a multifactorial framework in which a variety of lifestyle factors or pharmacological treatments are measured in conjunction with physical activity and diet. Professionals carrying out such programs and interventions often exclude or simply overlook the role of medication on weight and lifestyle. Therefore, the Canadian Institute for Health Information ${ }^{23}$ recommends that an expert or pharmacist be integrated into the team of professionals to address this salient and frequently contributing factor. Future intervention research is warranted to test this proposed multidisciplinary approach to help researchers and clinicians develop suitable interventions and preventative measures to reduce, or at the very least, delay decline in mobility and the onset of cognitive impairment and dementia in obese seniors thus ultimately prolonging independent living and quality of life.

\section{Acknowledgements}

None.

\section{Conflict of interest}

The author declares no conflict of interest.

\section{References}

1. United States Census Bureau. International programs. 2014.

2. Statistic Canada. Data Products-2016 Census. 2017.

3. Statistic Canada. Population count and population growth in Canada. 2015.

4. Flegal KM, Kruszon Moran D, Carroll MD, et al. Trends in obesity among adults in the United States, 2005 to 2014. JAMA. 2016;315(21):2284 2291.

5. Yang L, Colditz GA. Prevalence of overweight and obesity in the United States, 2007-2012. JAMA Inter Med. 2015;175(8):1412-1413.
6. Baker A, Sirois Leclerc H, Tulloch H. The impact of long-term physical activity interventions for overweight/obese postmenopausal women on adiposity indicators, physical capacity, and mental health outcomes: A systematic review. J Obes. 2016;2016:6169890.

7. Lau DC, Douketis JD, Morrison KM, et al. 2006 Canadian clinical practice guidelines on the management and prevention of obesity in adults and children [summary]. CMAJ. 2007;176(8):S1-S13.

8. Rejeski WJ, Marsh AP, Chmelo E, et al. Obesity, intentional weight loss and physical disability in older adults. Obes Rev. 2011;11(9):671-685.

9. Jensen G, Hsiao PY. Obesity in older adults: Relationship to functional limitation. Curr Opin Clin Nutr Metab Care. 2010;13(1):46-51

10. Segev Jacubovski O, Herman T, Yogev Seligmann G, et al. The interplay between gait, falls and cognition: can cognitive therapy reduce fall risk? Expert rev neurother. 2011;11(7):1057-1075.

11. Verghese J, Robbins M, Holtzer R, et al. Gait dysfunction in mild cognitive impairment syndromes. J Am Geriatr Soc. 2008;56(7):1244 1251 .

12. Pietrzykowska NB. Obesity in the Elderly. USA: Obesity Action Coalition (OAC); 2016

13. Lang IA, Llewellyn DJ, Alexander K, et al. Obesity, physical function, and mortality in older adults. J Am Geriatr Soc. 2008;56(8):1474-1478.

14. Smith E, Hay P, Campbell L, et al. A review of the association between obesity and cognitive function across the lifespan: Implications for novel approaches to prevention and treatment. Obes Rev. 2011;12(9):740-755

15. Fitzpatrick AL, Kuller LH, Lopez OL, et al. Midlife and late-life obesity and the risk of dementia. Arch Neurol. 2009;66(3):336-342.

16. Villareal DT, Chode S, Parimi N, et al. Weight loss, exercise, or both and physical function in obese older adults. $N$ Engl $\mathrm{J}$ Med 2011;364(13):1218-1229.

17. Starr KNP, Mc Donald SR, Bales CW. Obesity and physical frailty in older adults: A scoping review of lifestyle intervention trials. $J$ Am Med Dir Assoc. 2014;15(4):240-250.

18. Lautenschlager NT, Cox KL, Flicker L, et al. Effect of physical activity on cognitive function in older adults at risk for Alzheimer disease: a randomized trial. JAMA. 2008;300(9):1027-1037.

19. Johns DJ, Hartmann-Boyce J, Jebb SA, et al. Diet or exercise interventions vs combined behavioral weight management programs:A systematic review and meta-analysis of direct comparisons. J Acad Nutr Diet. 2014;114(10):1557-1568.

20. Kramer AF, Erickson KI. Capitalizing on cortical plasticity: Influence of physical activity on cognition and brain function. Trends $\operatorname{Cogn} \mathrm{Sci}$. 2007;11(8):342-348

21. Hamdy O. Obesity treatment and management. USA: Medscape; 2017.

22. Moran M, Van Cauwenberg J, Hercky-Linnewiel R, et al. Understanding the relationships between the physical environment and physical activity in older adults: A systematic review of qualitative studies. Int $J$ Behav Nutr Phys Act. 2014;11(1):79-91.

23. Canadian Institute for Health Information. Health care in Canada, 2011: A focus on seniors and ageing. USA; 2011 